\title{
Granulation tissue-derived mesenchymal stromal cells: a potential application for burn wound healing in pediatric patients
}

\author{
Pelizzo $\mathrm{G}^{1}$, Avanzini $\mathrm{MA}^{2}$, Mantelli $\mathrm{M}^{2}$, Croce $\mathrm{S}^{2}$, Maltese $\mathrm{A}^{2}$, Vestri $\mathrm{E}^{3}$, De Silvestri $\mathrm{A}^{4}$, Percivalle $\mathrm{E}^{5}$, Calcaterra $\mathrm{V}^{6}$
}

\begin{abstract}
Objective: Multipotential cells are mobilized into peripheral blood in response to trauma, in particular in severe burns. These cells migrate to the site of injury in response to chemotactic signals to modulate inflammation, repair damaged tissue and facilitate tissue regeneration. We evaluated the possibility of isolating and in vitro expand mesenchymal stromal cells (MSCs) from granulation tissue (GT) during debridement of a burn wound, as a persective strategy to improve skin regeneration.

Methods: GT obtained from a 12-month-old burn patient was in vitro cultured. Expanded MCSs were characterized for morphology, immunophenotype, differentiation capacity and proliferative growth. Antifibrotic features were also evaluated.

Results: It was possible to isolate and in vitro expand cells from GT with the morphology, phenotype, proliferative and differentiation capacity typical of MSC, these cells were defined as GT-MSC. GT-MSCs exhibited antifibrotic features by releasing soluble factors, this activity was superior to that observed in BM-MSC.

Conclusions: Successful isolation and expansion of MSCs from GT is reported. Considering their functional characteristics, GT-MSCs could be considered a good candidate adjuvant therapy to improve burn wound healing, particularly in pediatrics.
\end{abstract}

Key Words: Mesenchymal stromal cells; Granulation tissue; Children; Burn; Wound regeneration

\section{Introduction}

Pediatric burns are a major form of injury, affecting millions of children worldwide, and may be caused by scald incidents, fire injury or child abuse ${ }^{[1]}$. Burn scars often create great functional problems and their disfigurement can cause serious psychosocial problems ${ }^{[2-4]}$.

Mesenchymal stromal cells (MSCs) have shown promise as a tool in cell therapy to treat different conditions, including wound regeneration ${ }^{[5-10]}$. MSCs are an outstanding tool for cell therapy applications, not only because of their multipotent nature, but also due to their ability to home to and engraft in damaged tissues, release trophic factors, promote neovascularization, manage oxidative stress and trigger anti-inflammatory responses ${ }^{[5,9-12]}$. Accumulating evidence suggests that MSC act through a combination of paracrine cell signalling and cell transdifferentiation, enhancing wound regeneration and improving angiogenesis $^{[12,}$ 13]. Recently, it has also been reported that multipotent cells mobilize to the peripheral blood after burn incidents and migrate to the site of injury in response to chemotactic signals where they modulate inflammation, repair damaged tissue and facilitate tissue regeneration ${ }^{[14,15]}$.

The most recognized source of MSCs is the bone marrow, however, other sources have been described such as adipose tissue, teeth, bone, muscle, placenta, liver, pancreas, umbilical cord and cord blood $^{[16]}$. Spyrou et $a{ }^{[17]}$. described the granulation tissue $(G T)$ as an abundant source of cells with important therapeutic efficacy in wound healing and tissue repair.

We describe the isolation and in vitro expansion of cells from granulation tissue (GT) obtained during debridement of a burn wound in a child. Based on these results, we propose that MSCs expanded from GT, may be considered a perspective strategy to improve skin regeneration in burn wound care.

\section{Methods}

GT was obtained from a 12 month-old boy undergoing burn treatment 15 days post-injury (severe thermal burn with full-thickness injury). The patient's GT was used for in vitro cell expansion, after obtaining written informed consent from parents.

\section{Isolation and culture}

Isolation and expansion of cells from GT, were performed following standard MSC culture procedures, as previously described $\left.{ }^{[18,} 19\right]$. Briefly, tissue samples were incubated at $37^{\circ} \mathrm{C}$ for 30 minutes, in serum-free $\alpha$-minimum essential medium ( $\alpha$ MEM) (Gibco, Life Technologies, Paisley, UK) supplemented with antibiotics and collagenase type II (Sigma Aldrich). Next, collagenase activity was blocked with complete medium $(\alpha \mathrm{MEM}+10 \% \mathrm{FBS}$, Euroclone, Milan, 
Italy). The cell suspension was then collected and plated at $160,000 / \mathrm{cm}^{2}$ in non-coated T175 flasks (Corning Costar, NY, USA) in D-MEM $+10 \%$ FBS and incubated at $37^{\circ} \mathrm{C}, 5 \% \mathrm{CO}_{2}$ for 48 -hours. Medium was then changed twice a week.

At confluence, MSCs were trypsinized (Trypsin EDTA, Lonza, Milan, Italy) and replated at 4,000 cells $/ \mathrm{cm}^{2}$, for expansion. Cells were propagated to reach senescence phase and kept in culture for additional 8 weeks.

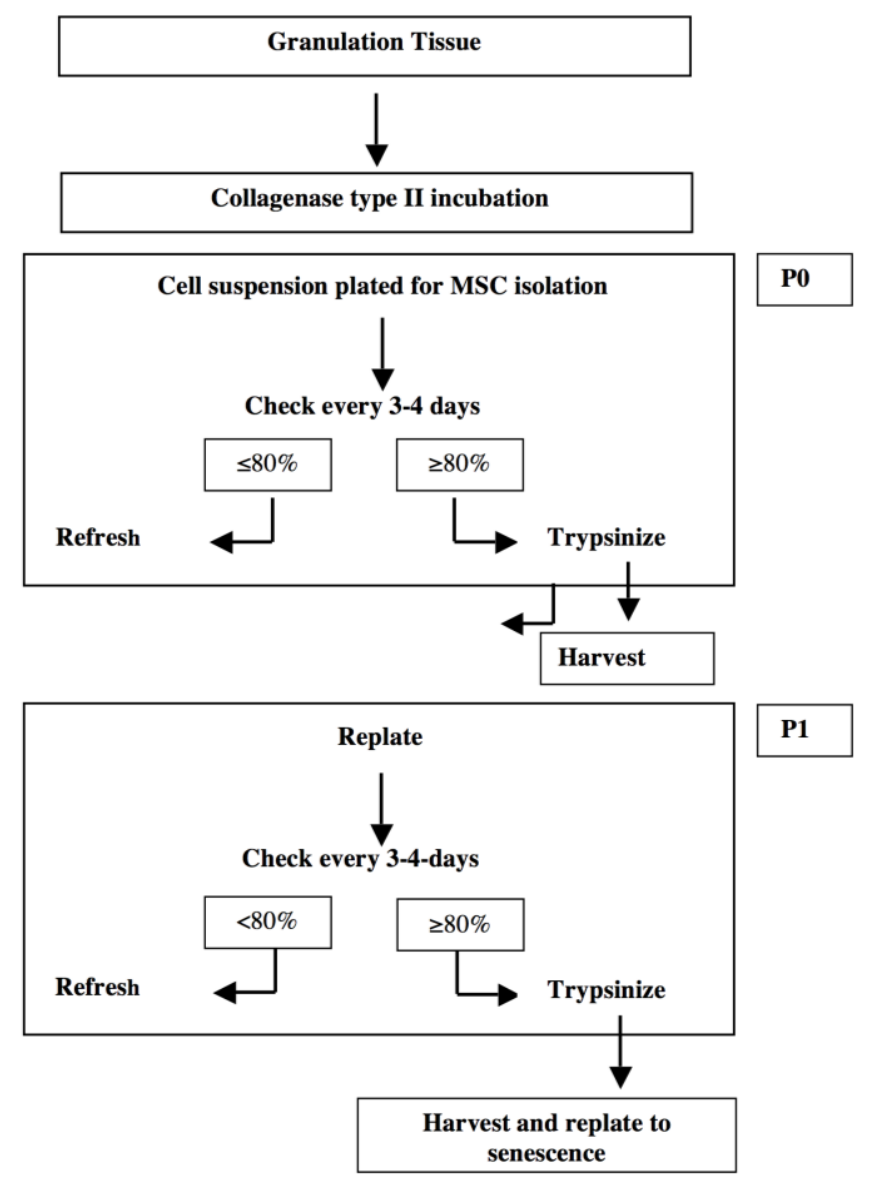

Figure 1. Scheme of the GT-MSCs isolation and expansion

Figure 1 illustrates the GT-MSCs isolation and expansion.

Characterization of ex-vivo expanded GT-cells

\section{Proliferative capacity}

Proliferative capacity was defined as cumulative Population Doubling (cPD) calculated with the following formula: $P D=\log$ ( $n$. of harvested cells/n. of plated cells) $/ \log 2$.

\section{Phenotyping}

Cells were characterized by flow-cytometry. Fluorescein isothiocyanate (FITC)- or phycoerythrin (PE)-conjugated monoclonal antibodies specific for CD73, CD34, CD90, CD14, CD45, CD31, CD105, class I-HLA and HLA-DR, (Beckman Coulter, IL, Milan, Italy) were used. Appropriate, isotype-matched, antibodies were employed as controls. Analysis was performed by direct immunofluorescence with a FACS Navios flow-cytometer (Beckman Coulter).

\section{Differentiation capacity}

The osteogenic and adipogenic differentiation capacity of GT-cells was assessed at P2-4 as previously described ${ }^{[18]}$. For osteogenic differentation complete medium supplemented with $10^{-7} \mathrm{M}$ dexamethasone and $50 \mathrm{mg} / \mathrm{ml} \mathrm{L}$-ascorbic acid and $5 \mathrm{mM} \mathrm{B-glycerol}$ phosphate (Sigma-Aldrich, St Louis, MO) was used while for adipogenic differentiation complete medium with $10^{-7} \mathrm{M}$ dexamethasone, $50 \mathrm{mg} / \mathrm{ml} \mathrm{L}$-ascorbic acid, $100 \mathrm{mg} / \mathrm{ml}$ insulin, 50 $\mathrm{mM}$ isobutyl methylxanthine, $0.5 \mathrm{mM}$ indomethacin (Sigma-Aldrich) and $5 \mathrm{mM}$ b-glycerol phosphate was used. After two week incubation, cells were stained for alkaline phosphatase (AP) activity using Fast Blue (Sigma-Aldrich) and for calcium deposition with Alizarin Red (Sigma-Aldrich) to evaluate osteogenic differentation. Adipogenic differentiation was assessed by staining of fat droplets awith Oil Red O (Sigma-Aldrich).

\section{Senescence assay}

Senescence was defined by $\beta$-galactosidase (SA- $\beta$-gal) staining Kit (Cell Signaling Technology, Danvers, MA), according to the manufacturer's instructions.

\section{Co-culture experiments}

To assess the paracrine effect of GT-cells, dermal fibroblasts were co-cultured using a transwell system. Briefly, GT-cells $\left(1.5 \times 10^{4}\right.$ cells/well) were plated on Transwell membrane (0.4-mm pores, Corning Costar) with fibroblasts $\left(6 \times 10^{4}\right.$ cells/well) in the lower chambers and cultured for 4 days in RPMI 1640 (Euroclone, Milano, Italy) $10 \%$ FCS (Euroclone). Co-cultures were stimulated with 100 $\mathrm{ng} / \mathrm{mL}$ of Fibroblast Growth Factor (FGF, Sigma, Milan, Italy) or not stimulated. For cell proliferation evaluation, fibroblasts were then harvested and viability was calculated using 0,2\% Trypan Blue (Sigma). Cultured fibroblasts alone were used as controls. In addition, the culture medium was collected at $72 \mathrm{~h}$ to examine the concentration of TGF $\beta$. Fibroblasts co-cultured with BM-MSCs were used as a control.

\section{TGF $\beta$ quantification by ELISA}

The quantification of TGF- $\beta 1$ levels in FGF activated fibroblast coculture supernatants were tested in order to define the role of this soluble factor in scar formation. Briefly, 96-well plates were coated with anti-human TGF- $\beta 1$ (Endogen Tema), in carbonate/bicarbonate buffer, $\mathrm{pH} 9.6$ overnight at room temperature. After three washes, a post-coating was performed for 1 hour at room temperature. After samples activation with $1 \mathrm{~N} \mathrm{HCl}$ for 10 minutes and neutralization with $1.2 \mathrm{~N} \mathrm{NaOH} / 0.5 \mathrm{M} \mathrm{HEPES}$, they were incubated for two hours at room temperature. Detection antibody, specific for TGF- $\beta 1$, was added and plate incubated at RT. After washing, Streptavidinconjugated horseradish peroxidase was added and plates were further incubated for 20 minutes at room temperature. Plates were washed again three times and substrate solution (hydrogen peroxide and stabilized tetramethylbenzidine) was added. After 20 minutes in the dark. Absorbance was read at $450 \mathrm{~nm}$ (Lettore Titertek Plus MS 212 - ICN). Cytokine concentrations (expressed in $\mathrm{pg} / \mathrm{ml}$ ) were calculated using the standard curve.

\section{Statistical analysis}

A comparison of the groups was made with Kruskall-wallis non parametric ANOVA followed by two- by-two subgroup analysis corrected for multiple tests. Statistical significance was defined as $\mathrm{p}<0.05$. Data analyses were performed with the STATA statistical package (released 14.2, 2012, Stata Corporation, College Station, Texas, USA). 
A

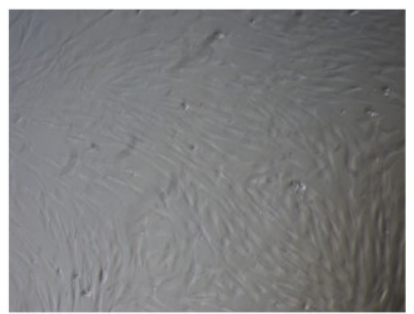

CD34

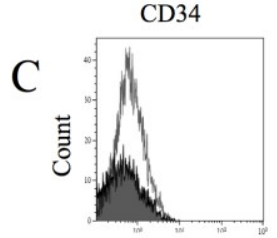

CD105
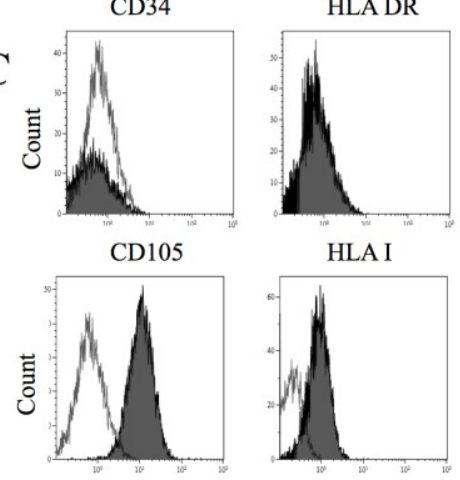

HLA I

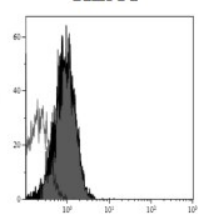

CD14

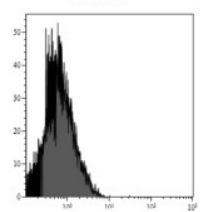

$\mathrm{CD} 90$

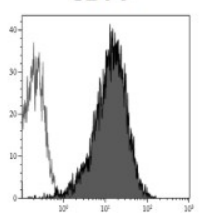

CD45

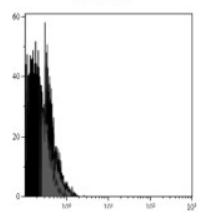

CD73

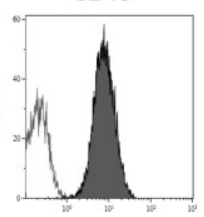

B

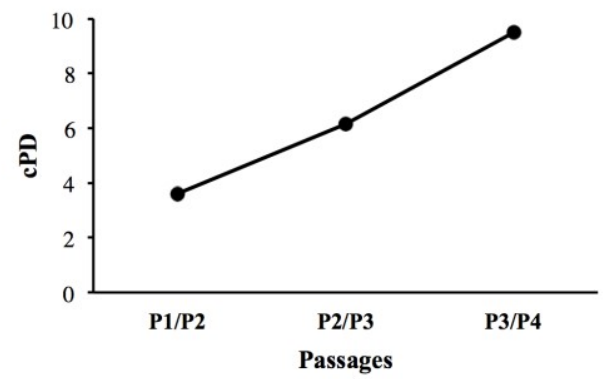

G

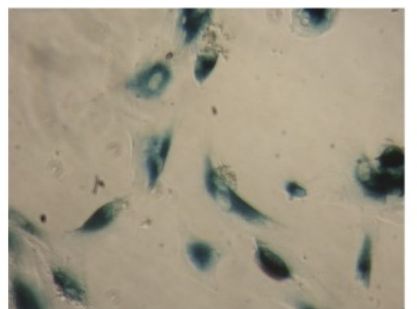

$\mathrm{D}$

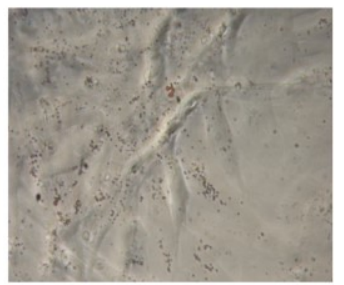

$\mathrm{E}$

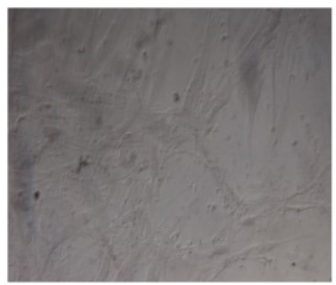

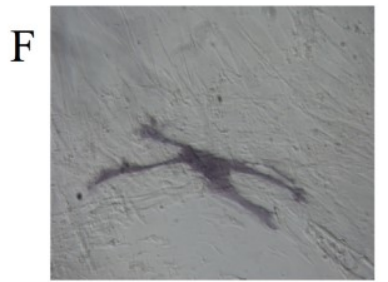

Figure 2. Characterization of GT derived cells. Panel A: typical "spindle-shape" morphology of in vitro expanded GT-MSCs, Original 10x magnification using an inverted Leica DM-IL microscope equipped with a Nikon Digital Sight DS-Fil camera linked to a NIS Element F Imaging system; Panel B: Proliferative capacity (defined as cumulative Population Doubling, $c P D$ ) was obtained by tracking cultured MSCS at each passage; Panel C: Representative surface antigen analysis by flow cytometry (y axis=cell numbers (count), $x$ axis = fluorescence intensity). Empty histogram represents isotype-matched control. Grey histogram represents stained cells. Overlapping histograms indicates absence of positive cells. Panel D-F: differentiation into adipocytes and osteoblasts. Poor differentiation capacity is revealed by the presence of small lipid droplet formation (stained with oil red O) and by poor calcium deposition (stained with alizarin red) and poor AP activity upon histological detection; Panel G: Representative evaluation of $\beta$-galactosidase activity in GT-MSC at senescence passage (P19). Senescent cells are stained blue.

\section{Results}

\section{Characterization of GT-derived cells}

Cells were successfully isolated from GT and propagated in vitro. After 7 days culture, it was possible to observe the appearance of 32 colony forming units (CFU-F) in $1,5 \times 10^{6}$ plated cells. Cells were plastic adherent and had the characteristic MSC "spindle-shape" morphology (Figure 2, Panel A) and normal proliferation capacity as demonstrated by cPD (Figure 2, Panel B). Moreover, they showed positive expression of CD73, CD90, CD105 and HLA-I and negative expression of CD34, CD14, CD45, and HLA-DR (Figure $2 \mathrm{C}$ ), the cells were termed GT-MSCs.

MSC isolated from GT poorly differentiated into adipocytes; this was confirmed by the presence of small lipid droplet formation (Figure 2D). MSC isolated from GT also poorly differentiated into osteoblasts, as confirmed by poor calcium deposition upon histological detection (Figure 2, Panel E); poor AP activity was noted as well (Figure 2, Panel F).
GT-MSCs were expanded up to P19, when they entered into senescence, as confirmed by typical senescence-associated $\beta$ Galactosidase staining (Figure 2, Panel G).

\section{Anti fibrotic effect of GT-MSC}

Fibroblasts cultured in the presence of GT-MSCs showed suppressed proliferation, (based on trypan blue viability score) compared with control fibroblasts both with or without inflammatory stimulation $(\mathrm{p}<0.001)$, while BM-MSCs did not induce this suppressive effect ( $p=0.19$ and $p=0.10$, respectively) (Figure 3, Panel A).

\section{TGF- $\beta 1$ quantification}

The quantification of TGF- $\beta 1$ levels in FGF activated fibroblast co-culture supernatants showed that in the presence of GT-MSC, TGF- $\beta 1$ levels were superimposable with that quantified in control wells $(1460 \mathrm{pg} / \mathrm{ml}$ and $1477 \mathrm{pg} / \mathrm{ml}$, respectively), while in the presence of BM-MSC, TGF- $\beta 1$ levels were slightly increased $(1651 \mathrm{pg} / \mathrm{ml})$ compared to controls $(\mathrm{p}=0.31)$ (Figure 3, Panel B). 


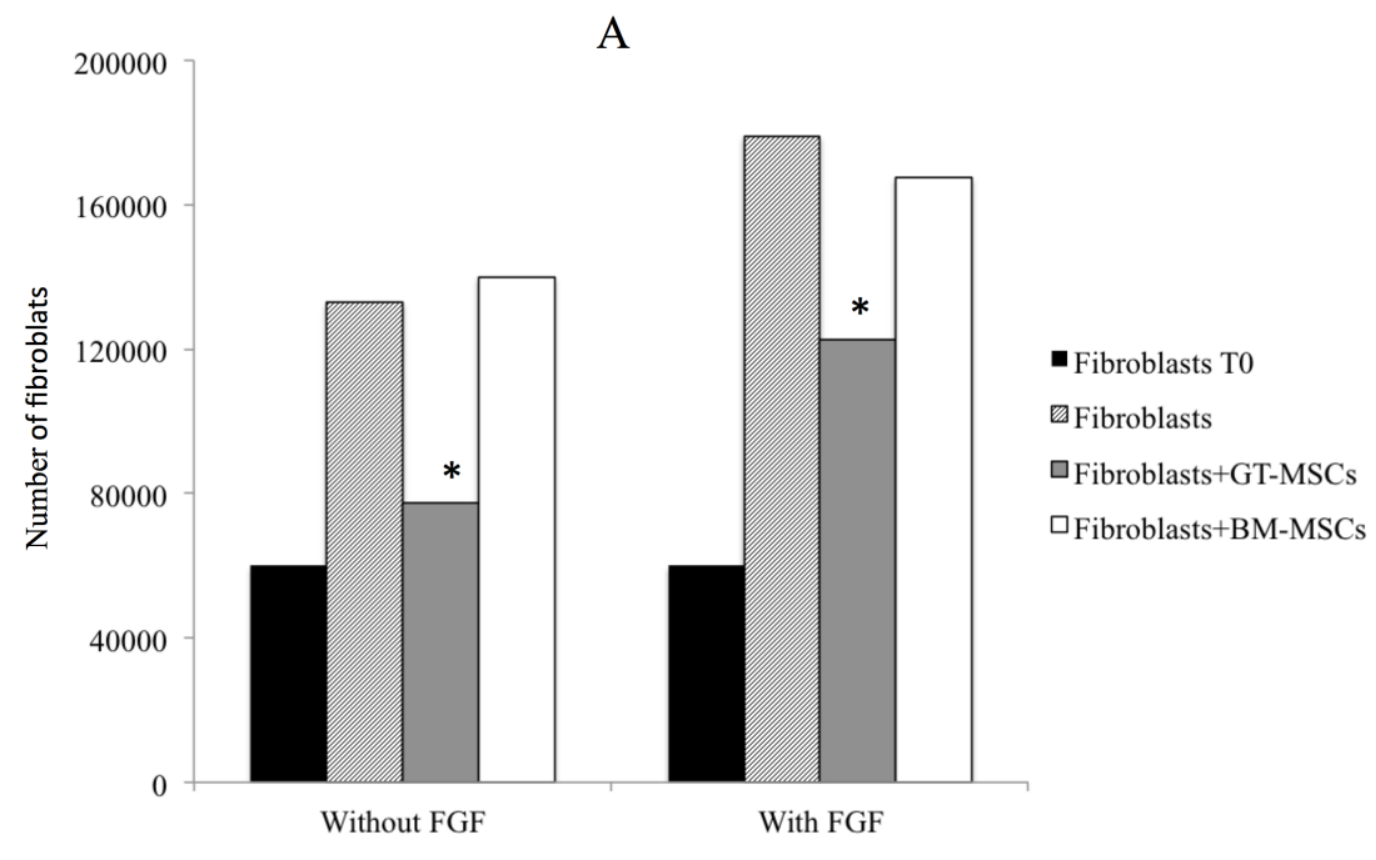

B

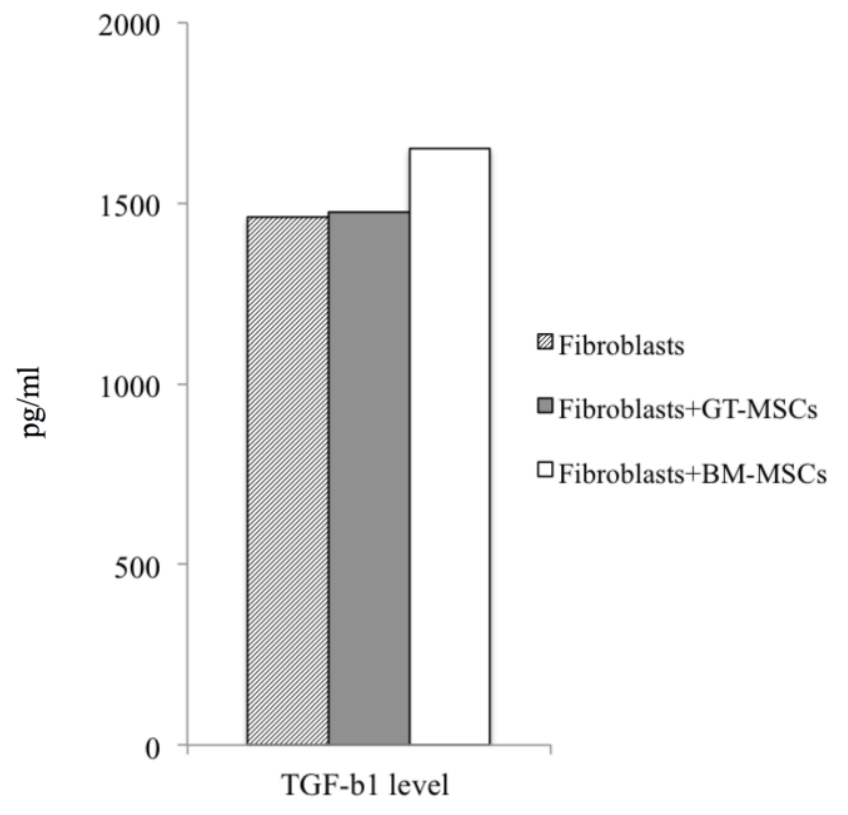

$* \mathrm{p}<0.05$ vs Fibroblasts

Figure 3. Anti fibrotic effect of GT-MSC and quantification of TGF-B1. Panel A: Number of fibroblasts alone (lined boxes) or in the presence of GT-MSCs (grey boxes) or BM-MSC

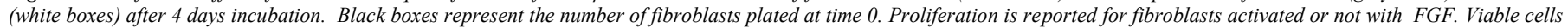

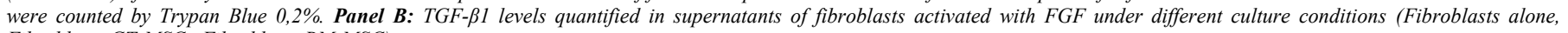
Fibroblast + GT-MSC, Fibroblast + BM-MSC).

\section{Discussion}

In this brief report we demonstrated that MSCs can be in vitro isolated and expanded from GT tissue of burn wounds. Cells derived from GT presented the typical MSC morphology and phenotype with regular proliferative capacity. GT-MSCs also exhibited antifibrotic features, for this reason they are an attractive alternative cell source with potential in burn wound healing and tissue repair.
The treatment of burns is a delicate art, especially in children and these injuries remain a major challenge worldwide. Nearly a fourth of all burn injuries occur in children under the age of 16, of whom the majority are under 5 years of age ${ }^{[1]}$. Scar tissue and skin grafts in children are subject to exagerated inflammatory responses, which may create a hypertrophic scar with an unsightly appearance and risk of impaired mobility ${ }^{[2-4]}$. To improve the quality of the scar, and diminish functional problems and disfigurement, new cell sources have been exploited for skin tissue regeneration. 
MSCs have also been proposed as therapy for lost tissues, including the skin, due to their potential to differentiate into multiple cell types and replace the missing tissue ${ }^{[5,9-12]}$. Although MSCs from bone marrow have been successfully used for therapeutic purposes and often designated as the gold standard, the accessibility and availability of these cells is limited, preventing their wider application in clinical situations. Therefore, more accessible stem cell sources have been proposed, such as peripheral blood, adult tissue such as adipose, teeth, bone, muscle, neonatal birthassociated tissues including placenta, cord blood and skin derivates $^{[16]}$.

To the best of our knowledge, we showed for the first time, that MSCs could also be isolated from debrided burn GT. Wound healing is a complex process that requires the interaction and coordination of a cascade of cellular responses to injury and includes three overlapping stages: inflammatory phase, fibroproliferative phase, and remodeling phase ${ }^{[20]}$. During the fibroproliferative stage, dermal fibroblasts from the wound margin proliferate and migrate into the wound, generate GT, and begin to remodel the wound matrix to create new dermal tissue. GT begins to be formed approximately four days after lesion formation, and is characterized by a high density of fibroblasts, granulocytes, macrophages and microcapillaries ${ }^{[20]}$. GT is often discarded during surgical burn treatment and thus represents a readily available and accessible source.

The first step in skin repair is wound closure, the next step is management of scar formation ${ }^{[20]}$. At this point, the skin has the capacity to stimulate the production of fibroblasts in very high quantities, especially in children, with the risk of creating a hypertrophic scar and consequent retraction or impaired articulation $^{[1-4]}$. There is increasing evidence that MSCs are trophic mediators of tissue repair; these cells promote scar-free wound healing and inhibit fibrotic tissue formation ${ }^{[21,22]}$. Paracrine effect is considered one of the main underlying mechanisms behind the therapeutic effects of stem cells ${ }^{[11,13,23]}$. In particular, the exosome, an essential paracrine factor for intercellular communication, plays a dominant role in cell-to-cell communication and modulates the molecular activities of recipient cells ${ }^{[9,11-13,23,24]}$. Our results confirm the anti-fibrotic properties of MSC, in particular GTMSCs. These features were demonstrated by in vitro suppression of fibroblast proliferation and inhibition of a the potent driver of tissue fibrosis, TGF- $\beta 1^{[25,26,27]}$. Moreover, we confirmed their paracrine method of action since results were obtained in co-culture experiments using transwell plates. Another interesting observation was the difference found between GT-MSC and BM-MSC in terms of antifibrotic activity; the former were more efficacious.

We also observed that adipogenic and osteogenic differentiation, hallmarks of MSCs, although detectable in all experiments, were less efficient in GT-MSCs compared with BM-MSCs, as demonstrated by microscopic examination. Indeed, contrasting data are available in the literature regarding the differentiation capacity of MSCs obtained from different sources ${ }^{[28-30]}$.

In conclusion, we reported on the successful isolation of MSCs from GT and their in vitro expansion. Although further studies are needed to confirm these observations, we speculate that GT-MSCs may be "committed" by the wound microenvironment and as such would be a logical candidate to improve burn wound management and skin repair, particularly in the pediatric setting.

\section{References}

1. Krishnamoorthy V, Ramaiah R, Bhananker SM. Pediatric burn injuries. Int J Crit Illn Inj Sci. 2012;2(3):128-34.

2. Louis MR, Meaike JD, Chamata E, Hollier LH. Practical considerations in pediatric surgery. Semin Plast Surg. 2016;30(4):171-75.

3. Bührer G, Beier JP, Horch RE, Arkudas A. Surgical treatment of burns: Special aspects of pediatric burns. Hautarzt 2017;68(5):385-92.

4. Shah AR, Liao LF. Pediatric burn care: Unique considerations in management. Clin Plast Surg. 2017;44(3):603-10.

5. Langrzyk A, Nowak WN, Stepniewski J, Jaźwa A, FlorczykSoluchU, Józkowicz A, Dulak J. Critical view on mesenchymal stromal cells in regenerative medicine. Antioxid Redox Signal. 2017. doi: 10.1089/ars.2017.7159.

6. Cerqueira MT, Marques AP, Reis RL. Using stem cells in skin regeneration: possibilities and reality. Stem Cells Dev. 2012;21(8):1201-14.

7. Cerqueira MT, Pirraco RP, Marques AP. Stem cells in skin wound healing: Are we there yet? Adv Wound Care (New Rochelle). 2016;5(4):164-75.

8. Keating A. Mesenchymal stromal cells: new directions. Cell Stem Cell. 2012;10(6):709-16.

9. Nuschke A. Activity of mesenchymal stem cells in therapies for chronic skin wound healing. Organogenesis. 2014;10(1):29-37.

10. Trohatou O, Roubelakis MG. Mesenchymal stem/stromal cells in regenerative medicine: Past, present, and future. Cell Reprogram. 2017;19(4):217-24.

11. Chen L, Tredget EE, Wu PY, Wu Y. Paracrine factors of mesenchymal stem cells recruit macrophages and endothelial lineage cells and enhance wound healing. 2008;3(4):e1886.

12. Vizoso FJ, Eiro N, Cid S, Schneider J, Perez-Fernandez R. Mesenchymal stem cell secretome: Toward cell-free therapeutic strategies in regenerative medicine. 2017;18(9).

13. Zhao B, Zhang Y, Han S, Zhang W, Zhou Q, Guan H, Liu J, Shi $\mathrm{J}, \mathrm{Su} \mathrm{L}, \mathrm{Hu} \mathrm{D}$. Exosomes derived from human amniotic epithelial cells accelerate wound healing and inhibit scar formation. 2017;48(2):121-13.

14. Rennert RC, Sorkin M, Garg RK, Gurtner GC. Stem cell recruitment after injury: lessons for regenerative medicine. 2012;7(6):833-50.

15. Ko IK, Lee SJ, Atala A, Yoo JJ. In situ tissue regeneration through host stem cell recruitment. 2013;45:e57.

16. Hass R, Kasper C, Böhm S, Jacobs R. Different populations and sources of human mesenchymal stem cells (MSC): A comparison of adult and neonatal tissue-derived MSC. Cell Commun Signal. 2011;9:12.

17. Spyrou GE, Watt DA and Naylor IL. The origin and mode of fibroblast migration and proliferation in granulation tissue. $\mathrm{Br} \mathrm{J}$ Plast Surg 1998;51(6):455-61.

18. Pelizzo G, Avanzini MA, Icaro Cornaglia A, Osti M, Romano P, Avolio L, Maccario R, Dominici M, De Silvestri A, Andreatta E, Costanzo F, Mantelli M, Ingo D, Piccinno S, Calcaterra V. Mesenchymal stromal cells for cutaneous wound healing in a rabbit model: pre-clinical study applicable in the pediatric surgical setting. 2015;13:219.

19. Camussi G, Deregibus MC, Bruno S, Cantaluppi V, Biancone L. Exosomes/microvesicles as a mechanism of cell-to-cell communication. 2010;78(9):838-48.

20. Gonzalez AC, Costa TF, Andrade ZA, Medrado AR. Wound healing - A literature review. An Bras Dermatol. 2016;91(5):614-620.

21. Li M, Luan F, Zhao Y, Hao H, Zhou Y, Han W, Fu X. Epithelial-mesenchymal transition: An emerging target in tissue fibrosis. Exp Biol Med (Maywood). 2016;241(1):1-13. 
22. Shen Q, Chen B, Xiao Z, Zhao L, Xu X, Wan X, Jin M, Dai J, Dai H. Paracrine factors from mesenchymal stem cells attenuate epithelial injury and lung fibrosis. Mol Med Rep. 2015;11(4):2831-7.

23. Stephen J, Bravo EL, Colligan D, Fraser AR, Petrik J, Campbell JD. Mesenchymal stromal cells as multifunctional cellular therapeutics - a potential role for extracellular vesicles. Transfus Apher Sci. 2016;55(1):62-9.

24. Fang F, Huang RL, Zheng Y, Liu M, Huo R. Bone marrow derived mesenchymal stem cells inhibit the proliferative and profibrotic phenotype of hypertrophic scar fibroblasts and keloid fibroblasts through paracrine signaling. J Dermatol Sci. 2016;83(2):95-105.

25. Pohlers D, Brenmoehl J, Löffler I, Müller CK, Leipner C, Schultze-Mosgau S, Stallmach A, Kinne RW, Wolf G. TGF-beta and fibrosis in different organs - molecular pathway imprints. Biochim Biophys Acta. 2009;1792(8):746-56.

26. Borthwick LA, Wynn TA, Fisher AJ. Cytokine mediated tissue fibrosis. Biochim Biophys Acta. 2013;1832(7):1049-60.
27. Huang S, Wu Y, Gao D, Fu X. Paracrine action of mesenchymal stromal cells delivered by microspheres contributes to cutaneous wound healing and prevents scar formation in mice. Cytotherapy, 2015;17(7):922-31.

28. Baksh D, Yao R, Tuan RS. Comparison of proliferative and multilineage differentiation potential of human mesenchymal stem cells derived from umbilical cord and bone marrow. Stem Cells. 2007;25(6):1384-92.

29. Bieback K, Kern S, Kocaömer A Ferlik K, Bugert P. Comparing mesenchymal stromal cells from different human tissues: bone marrow, adipose tissue and umbilical cord blood. Biomed Mater Eng 2008;18(1 Suppl):S71-6.

30. de Girolamo L, Lucarelli E, Alessandri G, Avanzini MA, Bernardo ME, Biagi E, Brini AT, D'Amico G, Fagioli F, Ferrero I, Locatelli F, Maccario R, Marazzi M, Parolini O, Pessina A, Torre ML, Italian Mesenchymal Stem Cell Group. Mesenchymal stem/stromal cells: a new "cells as drugs" paradigm. Efficacy and critical aspects in cell therapy. Curr Pharm Des. 2013;19(13):2459-73.

\section{Abbreviations}

$\begin{array}{ll}\text { AP } & \text { Alkaline phosphatase } \\ \text { CFU-F } & \text { Colony forming units } \\ \text { FITC } & \text { Fluorescein isothiocyanate } \\ \text { GT } & \text { Granulation tissue } \\ \text { MSCs } & \text { Mesenchymal stromal cells } \\ \text { PD } & \text { Population doubling } \\ \text { PE } & \text { Phycoerythrin } \\ \text { SA- } \beta \text {-gal } & \beta \text {-galactosidase } \\ \alpha \text { MEM } & \alpha \text {-minimum essential medium }\end{array}$

\section{Potential Conflicts of Interests}

None

\section{Sponsors/grants}

We thank Dr. E. Lenta for technical assistance and Dr. L. Kelly for English revision of the manuscript.

\section{Corresponding Author}

Gloria Pelizzo, Pediatric Surgery Unit, Children's Hospital, Via dei Benedettini n.1, 90134 Palermo, Italy; E-mail: gloriapelizzo@gmail.com 